\title{
La fantaisie et la nature des femmes dans l'Heptaméron de Marguerite de Navarre
}

MARIE CLAUDE MALENFANT

Résumé: Les occurrences de fantaisie dans l'Heptaméron de Marguerite de Navarre instaurent une différenciation entre les sexes des personnages du récit-cadre et des contes. Mais lorsqu'on considère la façon dont s'enflamme la fantaisie de l'un et l'autre sexe, les conséquences de cette flamme, puis la manière dont l'être peut et doit ensuite réagir, il apparaît que cette différenciation tient peut-être moins à la nature spécifique des sexes qu'à l'éthique et la pratique sociales qui leur imposent une nature différente.

Dour "faire conclusion du cueur de l'homme et de la femme," dit Simontault au devis de la $21^{\mathrm{e}}$ nouvelle, "le meilleur des deux n'en vault riens" (p. 175). ${ }^{1}$ D'autres devisants de l'Heptaméron se rangeront également à cette cruelle conclusion au cours de leur examen attentif des souffrances et fragilités de la condition humaine, ${ }^{2}$ car, au-delà de la polémique qu'entretiennent les personnages de la communauté de Serrance, avec la vivacité de jouteurs de la Querelle des Femmes, il est un principe unificateur qui semble justifier leurs prises de parole: ${ }^{3}$

les maulx que nous disons des hommes et des femmes [dit Oisille] ne sont poinct pour la honte particulliere de ceulx dont est fait le compte, mais pour oster l'estime de la confiance des creatures, en monstrant les miseres où ilz sont subjectz, afin que nostre espoir s'arreste et s'appuye à Celluy seul qui est parfaict et sans lequel tout homme n'est qu'imperfection (devis de la $48^{e}$ nv., p. 317). 
Mais il faut bien convenir que l'exposé de Marguerite de Navarre sur l'imperfection humaine se traduit en des termes différents selon que l'on soit homme ou femme. Les emplois du terme "fantaisie" indiquent comment les devisants, tout en déclarant leur intention de montrer que "les hommes et les femmes sont commungs aux vices et vertuz" (Ennasuitte, p. 263), ne peuvent faire abstraction des présupposés qui fondent une hétérogénéité sexuelle nécessairement hiérarchisée: ${ }^{4}$ la façon dont les conteurs et les personnages de leurs récits respectifs ressentent les émotions, puis jugent de l'action ou de la réaction qu'il convient d'entreprendre, actualisent ainsi l'épistémè de l'époque dont ils sont issus.

Au seizième siècle - et jusqu'au dix-huitième - le terme "fantaisie" désigne l'une des facultés de l'âme (comme doublet de l'imagination) de même que les résultats de l'application de cette faculté, résultats incluant aussi bien des représentations ou des productions reçues ou créées dans l'esprit, que des opinions, inclinations, ou désirs marqués par l'expression individuelle, qui peuvent être concurrents à la volonté et parfois même s'y soustraire. ${ }^{5}$ Selon la théorie aristotélicienne des fantasmes, les images captées par les sens extérieurs sont d'abord transmises au ventricule du sens commun d'où elles transitent vers celui de la faculté imaginative (ou fantaisie) qui trie et préforme en quelque sorte ces images avant qu'elles ne reçoivent le jugement de la raison et qu'elles n'aillent s'imprimer dans la mémoire — d'où l'imagination pourra toujours les rappeler à elle.

Or, selon les théories psychomédicales héritées de l'Antiquité, progressivement réévaluées au Moyen Âge et à la Renaissance, l'humidité et la froideur caractéristiques de la complexion féminine (définie par opposition au tempérament masculin, chaud et $\mathrm{sec}$ ) favorisent particulièrement les activités de la mémoire et de l'imagination. La faculté mémorative absorbe et retient les images un peu de la même manière que la cire retient la forme du cachet qui lui est imprimé: l'humidité et la froideur accrues du cerveau féminin participent alors à une meilleure rétention des images. Toutefois, les images perçues et créées par l'imagination féminine, parce que froides et humides, ne connaissent pas la stabilité et demeurent susceptibles de métamorphoses. Ainsi s'expliquent la versatilité du comportement féminin, ${ }^{6}$ de même que l'étrange pouvoir de la fantaisie des femmes enceintes dont l'activité est "si prodigieuse qu'elle étouffe toutes les autres facultés raisonnables," tandis que les fortes impressions conçues dans l'âme de la mère risquent d'être transmises au foetus et de se dupliquer servilement sur le corps de l'enfant ${ }^{8}$ qui naîtra difforme ou monstrueux. 
Alors même que les médecins de la Renaissance réévaluent, à la lumière des observations anatomiques, les doctrines aristotélicienne et galénique, ils sélectionnent et préservent néanmoins certains arguments confirmant l'infériorité et la faiblesse psychologique du tempérament féminin, ${ }^{9}$ même après avoir rejeté la thèse du mâle "blessé," "imparfait," voire de l'être "monstrueux"; ${ }^{10}$ historiquement, remarquait Évelyne Berriot-Salvadore, c'est au cours du seizième siècle que "l'irrascibilité de l'utérus se substitu[e] au tempérament humide pour expliquer l'infériorité" 11 de la femme, naturellement valétudinaire $^{12}$. Jean Liébaut précisait, par le titre même du premier chapitre de son Thresor des remedes secrets pour les maladies des Femmes que "la femme n'est animant mutile, ni imparfaict, mais foible \& maladif' (p. 2), car l'utérus, ce "terrible animal,"13 pour reprendre les termes de Rondibilis au Tiers Livre, "a colliguance a toutes les parties principales du corps." 14 Les humeurs d'une matrice insatiable ${ }^{15}$ — dont l'appétit se justifie sans doute par la croyance au "grand plaisir que prend la matrice à attirer, succer \& concevoir \& jouyr de la semence virile" (J. Liébaut, p. 61) - , les vapeurs de sang menstruel ou d'humeur spermatique non excrétées, s'élèvent alors jusqu'au foie, au coeur ou au cerveau, où elle peuvent causer frénésies, mélancolies, endormissements et corruption des facultés rationnelles. ${ }^{16}$ Ainsi la femme, bien qu'égale à l'homme en essence et en puissance, s'avère naturellement incapable de contrôler son corps et son esprit.

Évidemment, l'Heptaméron n'inscrit pas directement les théories portant sur la différenciation des sexes, mais leurs présupposés informent néanmoins tous les niveaux du récit. Lors du débat sur la $12^{\mathrm{e}}$ nouvelle, la narratrice ${ }^{17}$ mentionne que "les dames, selon leur coustume, parloient autant par passion que par raison" (p. 95); or, l'énoncé d'une faculté rationnelle ordinairement concurrencée par la passion ne trouve pas d'équivalent lors de descriptions de comportements masculins - on en déduit que ce n'est pas là "leur coustume." 18 De fait, cette concurrence dérive du présupposé de la prévalence de l'imagination chez la femme qui, à cause d'une complexion flegmatique ou d'un utérus influant sur ses facultés, conçoit de fortes et ardentes imaginations, provoquant des émotions extrêmes que la raison ne parvient pas à endiguer. ${ }^{19}$

Les 25 occurrences du mot "fantaisie," ${ }^{20}$ majoritairement présentes au niveau métadiégétique (dans les nouvelles ${ }^{21}$ ), réaffirment la différenciation des sexes: si certains emplois s'appliquent aussi bien à l'un et l'autre sexe, les occurrences inscrivant la fonction de la faculté imaginatrice dans le jugement concernent plus spécifiquement les femmes (personnages des récits et devisantes), tandis que les acceptions liées à l'inclination et au désir amoureux 
s'assimilent plus fréquemment aux hommes. Sur la base de cette première répartition, les emplois de "fantaisie" doivent encore être considérés selon la valeur de l'occurrence (positive, neutre ou péjorative), l'identité et le sexe de l'instance qui l'énonce, l'objet auquel elle s'applique, le contexte d'insertion (à l'égard des sentiments, opinions ou actions d'un personnage ou devisant, de sexe masculin ou féminin) et la relation de cette occurrence avec les thématiques narratives, ou encore, discursives, dans le cas des devis, ${ }^{22}$ dans lesquelles elle peut s'inscrire.

L'occurrence la plus neutre du recueil est introduite dès la situation initiale de la $18^{\mathrm{e}}$ nouvelle où un "jeune escolier" sera soumis à une double épreuve par celle qu'il aime. Hircan raconte la naissance de leur amour:

Vous, qui sçavez le prompt chemyn que faict ce feu quant il se prent à ung des boutz du cueur et de la fantaisie, vous jugerez bien que entre deux si parfaictz subjectz n'arresta gueres Amour, qu'il ne les eust à son commandement, et qu'il ne les rendist [. . .] si remplis de sa claire lumiere, que leur penser, vouloir et parler n'estoient que flambe de cest Amour (p. 138).

Ce "vous" à qui s'adresse Hircan renvoie ici spécifiquement à ceux et celles qui connaissent la fulgurance du sentiment amoureux, qui sont donc aptes à juger de l'ampleur de l'émotion et de son emprise sur la volonté individuelle. ${ }^{23}$ Une distinction de même nature avait été effectuée plus tôt par Geburon, dans la clausule du $16^{\mathrm{e}}$ récit, à propos d'amants qui s'étaient juré une fidélité éternelle:

Et, comme si la volunté de l'homme estoit immuable, se jurerent et promirent ce qui n'estoit en leur puissance: c'est une amityé perpetuelle, qui ne peult naistre ne demorer au cueur de l'homme; et celles seulles le sçavent, qui ont experimenté combien durent telles oppinions (p. 132-133).

$\mathrm{Si}$, selon Hircan, les deux sexes sont indifféremment susceptibles de connaître le foudroiement et l'aveuglement de la passion, ce sont les femmes qui, selon Géburon, peuvent le mieux juger de la brièveté de l'amitié "au cueur de l'homme." Simontault introduit par la suite une autre distinction entre les savoirs et expériences de l'un et l'autre sexes, alors que Parlamente et Longarine évaluent le degré de difficulté des épreuves de l'amant de la $18^{\mathrm{e}}$ nouvelle: "Vous en parlez bien à voz aises, dist Simontault, mais nous, qui sçavons que la chose vault, en debvons dire nostre oppinion (p. 141). ${ }^{24}$ Aux interlocutrices de Géburon, expérimentées quant à la valeur des opinions amoureuses des hommes, aux interlocuteurs expérimentés - mais 
indifférenciés - auxquels s'adressait Hircan, succède un "vous" féminin, qui se prononce sur la question mais à qui Simontault refuse la compétence de se prononcer, et un "nous" masculin, qui sait ce que la "chose" vaut ${ }^{25}$ — et qui ne vaut pas la peine de se soumettre à de telles épreuves. Géburon affirme que la femme sait bien que l'amour des hommes ne dure pas, ${ }^{26}$ mais Simontault insinue qu'elle ignore cependant pourquoi il en va ainsi, car elle ne juge pas de la "chose" de la même façon que les hommes qui demeurent les seuls à savoir ce qu'elle vaut. Parler d'amour et prononcer un jugement sur les faits d'amour font ainsi appel à des "savoirs" que ne partagent pas également tous les devisants, savoirs qui orientent leurs récits et colorent leurs devis. ${ }^{27}$

Michel Bideaux a relevé que l'on parlait, dans l'Heptaméron, plus abondamment d'aimer que d'amour, et que les 63 contes qui abordent ce thème distinguent deux façons d'aimer, traduites par les substantifs "amitié," lorsque le sentiment s' investit de valeurs positives, et "affection" ou "passion," quand il s'agit de décrire un comportement passif, pathologique ou criminel. ${ }^{28}$ Quand le feu d'amour "se prent à ung des boutz du cueur et de la fantaisie," les personnages amoureux des récits qui ne peuvent jouir de leurs désirs connaissent, effectivement, le versant le plus sombre de l'“affection," alors que la soumission de leur volonté à la passion et leur sollicitude démesurée à l'égard de l'être aimé évoquent les descriptions de l'amor hereos. ${ }^{29}$ Les symptômes de l'amor hereos l'assimilent, depuis les traductions et commentaires des traités de médecine arabe qui furent effectués au cours du Moyen $\hat{A g e}^{30}$, à une espèce particulière de mélancolie, ${ }^{31}$ caractérisée par la fixation de l'esprit sur l'image de l'objet d'amour, par la soumission de toute activité intellectuelle à la domination de cette image,$^{32}$ et par l'alternance de prostration et d'extrêmes variations émotives. ${ }^{33}$ La mélancolie érotique résulterait de la corruption de la faculté imaginative, ${ }^{34}$ ou encore, selon les termes des Questions problématiques du pourquoy d'amour, d' "un discord \& general divorce / D'entre le sens \& le vray jugement," $d$ '“ung travail d'ymagination [...]/Faisans les sens gouverneurs de raison." 35

Il est remarquable que, dans l'Heptaméron les occurrences de "fantaisie" entrent plus souvent en contact avec l'isotopie "fureur, mélancolie, désespoir et perte du sens" lorsque les récits sont énoncés par des femmes. ${ }^{36}$ Et même si, dans la série des dix récits inscrivant simultanément le terme "fantaisie" et les symptômes des affections de l'âme, les personnages masculins et féminins subissent ces désordres amoureu $\mathrm{x}^{37}$ dans des proportions égales, même si les destins des deux sexes sont plus fréquemment la mort ${ }^{38}$ et la réclusion, ${ }^{39}$ la répartition des affections diffère, ${ }^{40}$ et les raisons de leur émergence ne sont pas 
les mêmes selon que l'on soit homme ou femme. Fureur, mélancolie, désespoir et perte du sens procèdent, chez les hommes, de la concupiscence charnelle, de la frustration du désir, du dépit de se voir éconduit ou trompé par l'objet d'amour, et, en dernier lieu, de la mort d'un être aimé. Chez les femmes, elles accompagnent également la concupiscence, la frustration du désir, le dépit d'être éconduite ou trompée ainsi que le traumatisme de la mort de l'amant, mais elles se manifestent en outre à l' issue d'une lutte intérieure pour préserver son honneur, au moment de perdre sa réputation d'honnêteté, après avoir été abusée et déshonorée, pour avoir manqué à son honneur ou s'être rendue coupable d'une conduite déshonorante comme celle de la mère incestueuse de la $30^{\mathrm{e}}$ nouvelle.

Tableau des motifs d'affections selon le sexe de l'énonciateur et le sexe du personnage des nouvelles

Affections

communes

aux deux sexes

Affections des

personnages masculins

Devisants

Devisantes

$8,10,16$,

$23,50,59$

charnelle

frustration du

désir

dépit d'être

éconduit(e)

dépit d'être

trompé(e)

mort d'un

être aimé

(duc de

70,71
Affections des

personnages féminins

Devisants Devisantes

1,26

70

Hircan: 19, 10, 19, 21, 70 (Duchesse 35 de Bourgogne)

35

15 (délaissée par le mari), 64, 70 (Duchesse de Bourgogne)

10, 70 (Dame du

Vergier)

Bourgogne)

40,50 


\section{Tableau des motifs d'affections des personnages féminins} selon le sexe de l'énonciateur

\section{Motifs d'affections}

Devisants Devisantes

Lutte intérieure pour préserver l'honneur

Au moment de perdre sa réputation

Avoir été abusée et déshonorée

Avoir manqué à son honneur

Coupable d'une conduite déshonorante
16,26

43,60

14,72

21,23
70 (Duchesse de Bourgogne en danger "d'un refus déshonorable")

70 (Dame du Vergier)

Par ailleurs, le terme "fantaisie" n'est jamais qualifié par l'énonciateur qui l'introduit dans son récit, à l'exception de trois occurrences: l' amant de la $43^{\mathrm{e}}$ nouvelle fréquente Jambique qui, afin de préserver son "honneur" (p. 297), ne se laisse jamais reconnaître:

Et continuerent longuement ceste vie, sans ce qu'il s'apperceust jamays qui elle estoit: dont il entra en une grande fantaisye, pensant en luy-mesmes qui se povoit estre: car il ne pensoit poinct qu'il y eut femme au monde, qui ne voullut estre vue et aymée. Et se doubta que se fust quelque maling esperit, ayant oy dire à quelque sot prescheur que qui auroit veu le diable au visaige, l'on ne aymeroit jamais. En ceste doubte-là, se delibera de sçavoir qui estoit ceste-là qui luy faisoit si bonne chere [. . . ] (p. 298).

La "grande fantaisye" de l'amant indique ici un désir de connaître l'identité de celle qui se donne en se dissimulant ("grande fantaisye" de savoir) ou encore, elle désigne la fabrication d'une hypothèse sur la signification de faits étranges ("grande fantaisye" comme fabrication de sens) ${ }^{41}$ qui conduit à l'identification, ironique, d'une puissance trompeuse, puisque aucune femme de ce nom ne saurait jouir des plaisirs d'amour sans se laisser admirer. 
Les deux autres occurrences de "fantaisie" qualifiées par le narrateur surviennent dans la $35^{\mathrm{e}}$ nouvelle $\mathrm{e}^{42}$ où une dame de la ville de Pampelune, sous couvert d' "amour spirituelle" se consume bientôt de désir charnel pour un cordelier au point de "luy escripre l' amour qu'elle luy portoit le plus doulcement qu'elle peut pour le commencement." Rapidement, la dame prend l'habitude "de mander par lectres au prescheur sa furieuse fantaisye." Le mari, qui intercepte toutes les lettres, lui "feyt passer sa tentation" en empruntant les vêtements du cordelier et en battant si bien sa femme que celle-ci, lors d'une rencontre ultérieure avec l'objet de son désir, "commencea a crier comme femme hors du sens," prouvant hors de tout doute qu'elle était désormais "bien chastiée de sa folle fantaisie." Dans les nouvelles où des personnages (masculins et féminins) conçoivent quelque "fantaisie persistante," assimilable au désir, les "fureur, mélancolie, désespoir et perte du sens" résultent généralement du dépit et de la frustration; ${ }^{43}$ mais c'est chez une femme que la fureur accompagne la naissance du désir. ${ }^{44}$

Hircan, narrateur de ce récit, réitère donc le topos selon lequel la femme ne connaît que des émotions extrêmes, une réitération que nul autre devisant, ni femme ni homme, ne pose aussi franchement; Hircan demeure également le seul à insinuer aussi crûment, dans les devis, que le raisonnement d'un de ses "égaux" au jeu (Prologue, p. 10) repose sur une opinion extravagante. ${ }^{45}$ Parlamente avançait, dans le devis de la $21^{\mathrm{e}}$ nouvelle, que "souvent sont differenz les fardeaulx de l'homme et de la femme," puisque l' amour chez une femme est "fondée sur Dieu et sur honneur," tandis que l'"amour de la plupart des hommes de bien est [.. . ] fondée sur le plaisir." ${ }^{46}$ Hircan lui répond en ces termes:

Voylà doncques une raison [. . .] forgée sur vostre fantaisie, de vouloir soustenir que les femmes honnestes peuvent laisser honnestement l'amour des hommes, et non les hommes, [l'amour] des femmes, comme si leurs cueurs estoient differens; mais combien que les visaiges et habitz le soyent, si croy-je que les voluntez sont toutes pareilles, sinon d'autant que la malice la plus couverte est la pire (p. 175).

Saffredent attaque à son tour, moins durement mais plus insidieusement, la "fantaisie" de Parlamente qui admire l'héroïne de la $42^{\mathrm{e}}$ nouvelle, refusant de céder à celui qu'elle aimait "plus que sa vie, mais non pas plus que son honneur":

Ostez cette opinion de vostre fantaisye [dist Saffredent] ${ }^{47}$ et entendez d'où est venu ce terme d'honneur quant aux femmes, car peut estre que celles qui 
en parlent tant, ne sçavent pas l'invention de ce nom. [En fait, l'yprocrisie est le surnom d'honneur, érigé en une] si cruelle loy, que mesmes celes qui ayment parfaictement, dissimullent, estimant vertu estre vice; mais celles qui sont de bon entendement et de sain jugement, ne tumbent jamais en de telles erreurs [. . .] (p. 294). ${ }^{48}$

Les "raison" et "opinion" de Parlamente sur l" "honneur" des femmes sont désavouées parce qu'elles reposent sur une prémisse erronée (forgée sur une fantaisie), une prémisse qui aura pour incidence de fausser son jugement sur le sujet, car cette opinion biaise la fantaisie et le jugement s'opère alors sur des informations déviées. Mais ces deux hommes ont peut-être intérêt à ce que Parlamente révise sa conception de l'honneur féminin: Hircan, son mari, dont l'expérience des femmes ne fait aucun doute (voir par exemple la $9^{e}$ nv., p. 53), cherche à la convaincre que les hommes ne sont pas pires que les femmes, sinon que celles-ci dissimulent ce que ceux-là avouent franchement - sa conduite n'est donc pas condamnable; Saffredent, désirant fort probablement, selon les pénétrantes observations d'André Tournon, ${ }^{49}$ nouer une "intrigue" avec Parlamente, insiste sur le fait qu'il ne faut pas confondre l'hypocrisie avec l'honneur, d'autant plus que la lutte de l'amour et de l'honneur dans le coeur d'une femme peut provoquer, comme ill' a démontré dans la $26^{\text {e }}$ nouvelle, "une fièvre continue, causée d'un humeur mélancolique" (p. 217) ${ }^{50}$ qui mène, inexorablement, à la mort.

Parallèlement, les devisantes emploient le mot "fantaisie" dans des sens qui ne concernent pas le rôle de la faculté dans le processus du jugement: lorsqu'elles commentent les nouvelles, le terme renvoie exclusivement à l'expression de la volonté personnelle de personnages féminins; ${ }^{51}$ lorsqu'elles répliquent à leurs vis-à-vis masculins, la fantaisie recouvre le sens de volonté, d'inclination, de désir, ${ }^{52}$ comme lorsqu'Ennasuitte disqualifie un Saffredent vieillissant ${ }^{53}$ qui, dans son épilogue de la $3^{\mathrm{e}}$ nouvelle, expliquait aux dames comment:

quand vos mariz vous donnent des cornes de chevreux, vous [pouvez] leur en donnez de cerf." Ennasuite commencea à dire, en riant: "Si vous aimiez autant que autrefois vous avez faict, vous endureriez cornes aussi grandes que ung chesne, pour en randre une à vostre fantaisye; mais, maintenant que les cheveulx vous blanchissent, il est temps de donner trêve à voz desirs (p. 27).

Tant dans les nouvelles que dans les devis, les “fantaisies" de l'un et l'autre sexe recouvrent donc des acceptions communes (dont l'expression du désir), tandis que certaines applications demeurent connotées par le sexe de 
l'énonciateur: les devisantes relient d'elles-mêmes plus fréquemment dans leurs récits les "fantaisies" aux désordres de l'âme; mais ce sont les hommes qui établissent que la fureur s'empare d'une femme dès la naissance de l'amour, et qui soulignent les désordres du jugement d'une de leurs compagnes en insistant sur l'extravagance de ses "raison" et "opinion." 54

Ces positions diverses sur les conduites et "honneurs" de l'un et l'autre sexe déclinent ainsi la différenciation sexuelle en regard d'une nuance exprimée, entre autres indicateurs, par une conception de la "fantaisie" qui a plus à voir avec l'inclination et le désir amoureux chez les hommes, avec la faculté de raisonner et le jugement des femmes, quand leur "fantaisie" est obnubilée par le désir, la jalousie ou encore leur honneur. ${ }^{55}$ Mais l'on peut encore s' interroger, lorsque les devisants reprennent Parlamente sur ses raison et opinion, si ce phénomène dérive d'une conception propre à Marguerite de Navarre, ou s'il procède plutôt de l'ethos des personnages masculins qui énoncent, pour convaincre les dames, les arguments et les lieux communs de la Querelle des Femmes, dans lesquels puisent également les devisantes. Simontault, ${ }^{56}$ Saffredent ${ }^{57}$ et Hircan ${ }^{58}$ réactivent ponctuellement certains topoï du Roman de la Rose; mais la voix de Parlamente évoque par ailleurs dans le devis de la 57 ${ }^{\mathrm{e}}$ nouvelle celle de Christine de Pisan, et plus encore, celle de la Raison de la Cité des Dames: "pour vous escouter dire du mal de nous [dit Parlamente], noz oreilles n'en sentiront poinct de douleur, car nous sçavons ce qui en est"' (p. 356). ${ }^{59}$

Bien sûr, au-delà des "savoirs" et "expériences" que les devisants des deux sexes se prêtent ou se refusent, il se dégage un consensus pour convenir que c'est sans nul doute ce qu'amour met “en la fantaisie” des êtres (p. 386) - ces désirs, jalousies, fureurs et mélancolies - qui rapproche le plus l'homme et la femme, ${ }^{60}$ qui rend comparables leurs fragilités respectives. ${ }^{61}$ Mais il reste encore à déterminer si l'énoncé de la différenciation des sexes dans l'Heptaméron se justifie uniquement en regard des présupposés liés à la nature physiologique des êtres, ou si n'interviennent pas, ultimement, une éthique et une pratique sociales qui imposent aux sexes une "nature" différente. Alors que la théorie médicale accrédite la croyance selon laquelle la femme qui imagine ou qui désire peut donner naissance à un monstre, Parlamente affirme (au devis de la $43^{\text {e }}$ nouvelle) que la femme oublieuse du "fardeau" de son honneur (p. 174) lorsqu'elle s'accorde la jouissance de son désir, se dénature et atteint ainsi, en quelque sorte, des proportions monstrueuses: "celles qui [comme Jambique] sont vaincues en plaisir ne se doibvent plus nommer femmes, mais hommes, desquelz la fureur et la concupiscence augmente leur 
honneur" (p. 301). La victoire des désirs sur l'honneur ne rend pas la femme semblable à l'homme, mais elle fait plutôt, d'une femme, un homme, parce qu'en s'abandonnant à la fureur et à la concupiscence, elle efface les marques de l'honneur de son sexe — “c'est doulceur, patience, et chasteté." ${ }^{2}$ Et le péril est peut-être plus grand qu'il n'y paraît: si Géburon affirme d'un ton léger que "nostre gloire, nostre felicité et nostre contentement, c'est de vous [. . .] oster ce qui vous est plus cher que la vie" (p. 133. ${ }^{63}$ il n'en demeure pas moins qu'il n'y a que des femmes, parmi tous les personnages de l'Heptaméron, qui succombent à la fureur, à la mélancolie, au désespoir, ou qui perdent totalement l'usage de la raison au moment où elles risquent de perdre leur honneur. ${ }^{64}$

\section{Université Blaise-Pascal, Clermont-Ferrand}

\section{Notes}

1. Les citations de l'Heptaméron sont extraites de l'édition de Michel François (Paris, Garnier Frères, 1960). À moins d'avis contraire, c'est moi qui souligne. Veuillez noter que, dans les notes, "nouvelle" sera abrégé sous la forme "nv." lorsqu'il s'agit simplement d'indiquer un renvoi.

2. Voir Michel Bideaux, Marguerite de Navarre: "L'Heptaméron," de l'enquête au débat (Mont-de-Marsan, Éditions InterUniversitaires, 1992), p. 312.

3. Lyne Mondor développe une hypothèse stimulante - à laquelle j'aurais tendance à adhérer - en considérant que c'est l'examen de la notion d' "honneur" masculin et féminin qui constitue le principe justificateur de l'ensemble du recueil (cf. "La notion d'honneur dans l'Heptaméron de Marguerite de Navarre," Mémoire de maîtrise, Université McGill, août 1995).

4. Diane Desrosiers-Bonin a démontré, dans "Le Même et l'Autre dans deux recueils de nouvelles de la Renaissance française" Carrefour, XVII, 2 (1995), 86-97), comment "il était encore impensable en cette première moitié du XVIe siècle de concevoir l'égalité dans la différence" (p. 94). À partir des récits de Marguerite de Navarre et de Jeanne Flore, il apparaît que, dans la première moitié du seizième siècle, les catégories du "Beau," du "Noble" et du "Vertueux" sont informées par "une vision hiérarchique du monde établie en fonction des paramètres du Même et où la différence [. . . et définie dans la contradiction, l'opposition, voire la subordination au Même" (p. 87).

5. Voir les actes des journées d'études sur la notion de "fantaisie" à la Renaissance, organisées par le groupe equil xvi (24 juin 1994 et 22-23 juin 1995) sous la direction de Marie-Luce Demonet (Université Blaise-Pascal, Clermont-Ferrand II), parus dans la revue électronique Renaissance Interactive (URL: http://odalix.univ-bpclermont.fr).

6. I. Maclean, The Renaissance Notion of Woman: A Study in the Fortunes of Scholasticism and Medical Science in European Intellectual Life (Cambridge, Cambridge University Press, 1979), p. 42. 
7. Évelyne Berriot-Salvadore, Un corps, un destin: la femme dans la médecine de la Renaissance (Paris, Champion, 1993), p. 132.

8. D'après l'étude de M.- H. Huet, les monstres apparaissent bien, en effet, comme une "traduction littérale" de l'image perçue par la femme sur la forme du foetus, sans interprétation et sans médiation, sans abstraction de la forme perçue à sa signification: le produit monstrueux résulte d'une reproduction mimétique et révèle ainsi la violence des désirs ou des peurs de la mère (Monstrous Imagination [Cambridge, Harvard University Press, 1993], pp. 26-27). Sur la notion de monstruosité à la Renaissance, voir l'étude de Jean Céard, La nature et les prodiges: l'insolite auXVI' siècle (Genève, Droz, 1977), spécialement les première et deuxième parties, pp. 1-158.

9. I. Maclean, pp. 35 et $44-45$.

10. Voir M. A. Zimara, Problèmes d'Aristote \& autres filozophes \& Medecins. .., trad. par Georges de La Bouthière (Lyon, J. de Tournes, 1554), pp. 108-110 et 140; J. Liébaut, Thresor des remedes secrets pour les maladies des Femmes, Traduict de Latin en François par M. I. Liebaut docteur en Medecine, Derniere edition, reueuë et corrigee (Paris, Estienne Valet, 1597), pp. 1-2.

11. Évelyne Berriot-Salvadore, Histoire des femmes en Occident (Paris, Plon, 1991), t. III, p. 370.

12. É. Berriot-Salvadore, Op. cit., 1993, p. 44.

13. Le Timée de Platon (éd. Émile Chambry, Garnier-Flammarion, 1969,91b) précise cependant qu'un tel animal avide de procréation existe chez l'homme également, mais les auteurs renaissants qui utilisent ce passage pour définir la nature de la femme ne le citent jamais complètement. Pour quelques occurrences de ce topos de la nature de la femme, voir l'Éloge de la Folie (Érasme, Oeuvres choisies, éd. Chomarat, [Paris, Librairie Générale Française, 1991], p. 128), le Tiers Livre de Rabelais (ch. XXXII, p. 357), et Alector ou le Coq de Barthélémy Aneau (éd. M.-M. Fontaine [Genève, Droz, 1996], t. I, chap. IX, p. 67).

14. François Rabelais, Tiers Livre, édition critique avec variantes, notes et intoduction établie par Jean Céard (Paris, Librairie Générale Française, 1995), chap. 23, p. 311.

15. C'est en vertu de cette assimilation que Bonnacciuoli faisait dériver étymologiquement le mot "vulve" de "volens" (I. Maclean, p. 40); la voracité de l'appétit utérin dériverait du principe aristotélicien selon lequel l'imperfection tend vers la perfection: "the comparison is found in Physics 1.9. [199a 22]: 'matter desires form as the female the male'" (idem). La tradition antiféminine s'appuie également sur l'autorité des Proverbes qui mentionnent la matrice au nombre des choses insatiables $(30,16)$.

16. Jean Liébaut explique que la rétention ou la superfluité de l'humeur spermatique et du sang menstruel peuvent rendre la matrice "valetudinaire \& propre a estre griefvement offensée," provoquant chez la femme "manies, resveries, fureurs de matrice, tres-longs sommeils, estonnemens, mouvemens desordonnez, pales couleurs," et chez la jeune vierge une dépravation du cerveau et de "la ratiocination, dont surviennent diverses sortes de resveries" (pp. 6-7 et 23).

17. Ou plutôt, l'instance d'énonciation première, narrateur extra et hétérodiégétique. 
18. En revanche, l'instance d'énonciation première intervient, au devis de la $40^{\circ}$ nv. (p. 280 ), en insinuant la duplicité des hommes à l'égard de leurs femmes: "Hircan, Geburon, Simontault et Saffredent jurerent qu'ilz s'estoient mariez en pareille intention [désireux de vivre en l'état de mariage comme Dieu et Nature l'ordonnent] et que jamais ilz ne s'en estoient repentis; mais quoy qu'il en fust de la verité, celles à qui il touchoit en furent si contantes [... .]."

19. Cf. I. Maclean, pp. 7, 16 et 42.

20. 19 par rapport à 7 dans les devis. Je laisserai ici l'occurence dérivée "mary fantasticque" (26" nv., équivalent de jaloux) ainsi que celle du sommaire de la $59^{\mathrm{c}}$ nv. qui fut ajoutée ultérieurement au texte de l'Heptaméron ("Cette mesme dame [. . .] par ce moyen, vecut depuis à sa fantasye"). Notons l'hétérographie du terme dans l'édition François: les deux graphies les plus fréquentes sont fantaisie (12) et fantaysie (12) - sans que l'on puisse en déduire une intention de signification - et l'on trouve une faintasie ( $10^{c} \mathrm{nv}$.) en plus de l'occurence de fantasye déjà mentionnée ( $59^{e} \mathrm{nv}$.). Remarquons enfin que ces occurrences recouvrent l'ensemble des acceptions de la notion de fantaisie, à l'exception toutefois des activités de réminiscence et de création artistique.

21. Nous considérerons, selon la terminologie de Genette (Figures III [Paris, Seuil, 1972], pp. 238-243), que la diégèse définit le niveau du cadre narratif (où l'instance narrative extradiégétique relate le récit de la rencontre des devisants, un récit d'action et de parole), alors que les devisants, instances intradiégétiques produisant eux-mêmes des récits, édifient le niveau métadiégétique de l'oeuvre: les contes y paraissent à titre de récits au second degré assumés, cette fois, par l'un ou l'autre des devisants.

22. Par considération de l'un ou l'autre des faisceaux de commentaires structurant les échanges.

23. Simontault semble cependant exclure Dagoucin de la catégorie des amoureux expérimentés (devis de la $8^{c}$ nv., p. 48).

24. Pour une construction parallèle, voir Ennasuitte répliquant à Hircan (p. 128): "Vous en parlez bien à vostre aise [. . .]; mais vous ne sçavez quel crevecueur c'est quant l'on ayme sans estre aymé?"

25. La "chose" pouvant concerner ici aussi bien les motifs de la femme qui "éprouve" son amant que la finalité de l'entreprise.

26. Mais, précise Parlamente, la femme "expérimentée seulement," car "il n'est rien plus sot, ne plus aysé à tromper, que une femme qui n’a jamais aymé” (devis de la 35 nv., p. 260).

27. Mentionnons un autre cas où le narrateur vise clairement un groupe de destinataires spécifiques: Dagoucin, en terminant son premier récit $\left(9^{e} \mathrm{nv}.\right)$, s'adresse aux devisants masculins - et il s'agit là de l'unique exemple, dans tout le recueil, d'une nouvelle destinée en épilogue aux hommes de la compagnie -, et plus particulièrement à Saffredent (affirmant au devis de la $8^{\mathrm{c}} \mathrm{nv}$. n'avoir jamais vu un homme mourir d'amour): "Que vous semble-t-il, Messieurs, qui n'avez voulu croyre à ma parole, que cest exemple ne soit pas suffisant pour vous faire confesser que parfaicte amour mene les gens à la mort, par trop celée et mescongneue" (p. 53).

28. Pp. 175 et 179. 
29. Cette forme désordonnée du sentiment amoureux résulte de la corruption de la vertu imaginative caractérisée par une sollicitude démesurée à l'égard de l'être aimé; les termes amor hereos sont attestés dans la traduction, par Constantin l'Africain, du Viaticum de Ibn Eddjezzar (D. A. Beecher et M. Ciavolella, A Treatise of Lovesickness, édition critique et traduction du traité De la maladie d'amour ou melancholie erotique [\{Paris, 1623\}, Syracuse, Syracuse University Press, 1990], p. 670. Pour une analyse des quatre "systèmes médico-philosophiques," parfois contradictoires, qui coexistent simultanément dans les définitions de l'amor hereos, de l'Antiquité à la fin du seizième siècle, voir l'article de D. A. Beecher, "L'amour et le corps: les maladies érotiques et la pathologie à la Renaissance," dans Le corps à la Renaissance. Actes du XXXe Colloque de Tours 1987 (Paris, Aux Amateurs de Livres, 1990), pp. 423-434.

30. Sur l'historique de l'assimilation pré-arabique du sentiment amoureux (désordre de l'âme) avec la mélancolie (désordre du corps), voir D. A. Beecher et M. Ciavolella, pp. 60-62; dès Hippocrate, la description des symptomes de la maladie d'amour assimile celle-ci à la mélancolie; mais dans les termes, seul le physicien Alexandre de Tralles (physicien de l'école de Byzance à Rome au sixième siècle) diagnostique clairement l'état dépressif d'une femme en l'absence de son mari comme un véritable cas de mélancolie. La doctrine de la "mélancolieérotique" dérive des traductions de traités des médecins arabes, et particulièrement des Practica Pantegni de Constantin l'Africain qui "posent un rapport entre l'amour (généralement appelé "hereos" et associé à la notion d'héroïsme) et la mélancolie; la passion y est, en fait, traitée comme une "species melancholiae - un type de mélancolie," et c'est là une notion dont l'importance sera déterminante pour la constitution du type littéraire de 1'“amant mélancolique" (R. Klibansky, E. Panofsky et F. Saxl, Saturne et la Mélancolie (Paris, Gallimard, 1989), p. 149). Pour un historique de l'étymologie de hereos, voir D. A. Beecher et M. Ciavolella, pp. 75-76.

31. J.L. Lowes, “The Loveres Maladye of Hereos," Modern Philology, XI, 1913-1914 (pp. 491 546), pp. 509-510.

32. La mélancolie érotique demeure étroitement liée à la manie, selon Constantin l'Africain (Breviarium Constantini dictum viaticum [Lyon, 1510], I, II), dans la mesure où les superfluités d'humeurs dont elles procèdent toutes deux se logent dans les cellules antérieures et médianes du cerveau et soumettent toute activité de l'esprit (J.L. Lowes,, pp. 515 et 506).

33. Questions problematiques du pourquoy d'Amours, nouvellement traduict, d'Italien en langue francoyse par Nicolas Leonique (Paris, Alain Lotrian, 1543), $\mathrm{f}^{\circ} \mathrm{A} 7^{\mathrm{v}}-\mathrm{A} 8^{\mathrm{r}}$ : " $L$ 'entendement \& fantasie de la personne [amoureuse], s'esmeut facillement par soubdaine vision de quelque chose qu'il desire, tellement que alors se pert $\&$ demeure suspens de ce qu'il doibt faire ou penser." Par ailleurs, l' amoureux est sujet à "loubliance," "à cause de la vehemente \& desordonnée commotion du sang \& des espritz autour du cueur [. . .] dont ce faict que les images imprimées incontinent sont effacées \& perdues, parquoy ne leur peut souvenir de ce qu'ilz avoient proposé de dire." Ce traité énumère, parmi les signes extérieurs des variations émotives extrêmes, la rougeur et la pâleur, les pleurs fréquents, la voix faible et le corps qui tremble, le vieillissement prématuré. Ultimement, l' amant peut même mourir de joie car "quant vient au jouyr de sa tant desirée partie toute la chaleur \& esprit naturel sortisse avec telle impetuosité es parties loingtaignes qu'il ne restast riens au dedans par 
quoy s'ensuyvroit mort subite. C'est cy la cause pourquoy plusieurs se sont esvanouyz en embrassant leur amyes: \& aucuns y sont mors" (B5 $\left.{ }^{\vee}\right)$. La $9^{\bullet} \mathrm{nv}$. de l'Heptaméron en donne une illustration: le jeune homme amoureux "fut habandonné de toutes ses vertuz et esperitz; car la joye les feit tellement dilater que le siege de l'ame luy faillyt, et s'envolla à son Createur" (p. 52).

34. J.L. Lowes, pp. 505-506; D.A. Beecher et M. Ciavolella, pp. 69 et 71.

35. “Qu'est-ce d'Amour" de Melin de Saint-Gelais, dans les Questions problematiques, $\mathrm{f}^{\circ} \mathrm{B} 7$.

36. Parmi les 38 nouvelles ou devis concernant les personnages des contes où se rencontre cette isotopie, nous relevons 9 occurences de "fantasie" dans les récits produits par des femmes et 5 par des hommes: les co-occurrences signifiantes (où la fantasie est liée aux affections de l'âme) demeurent plus nombreuses chez les femmes ( 5 contre 2 chez les devisants masculins en contexte élargi, et 3 contre 1, directement liées à "fantaisie": Ennasuitte, nv. 19, Longarine, nv. 50, Parlamente, nv. 64, Hircan, nv. 35). Sur l'ensemble des 38 récits qui forment cette isotopie, les instances d'énonciations féminines sont légèrement plus nombreusess (26 interventions masculines et 30 féminines) alors que les devisantes (essentiellement Parlamente, Oisille et Longarine, Ennasuitte dans une moindre mesure mais aucune intervention émise par Nomerfide) assimilent plus fréquemment les affections de l'âme aux comportements masculins: 20 prises de parole féminines mentionnent 32 affections (surtout la fureur) affligeant des hommes, mais 9 seulement se distribuent les 18 occurrences (dont 13 sous les catégories du désespoir et de la perte du sens) qualifiant des conduites féminines.

37. Les affections surviennent essentiellement des suites d'amours malheureuses, hormis dans les cas de la nv. 51 (fureur subséquente à la colère) et de la nv. 70 (mouvements de fureur inspirés par la frustration du désir de connaître un secret). On note également des exceptions chez les personnages féminins, dans la $22^{\mathrm{e}} \mathrm{nv}$. (désespoir de la mère provoqué par le déshonneur de sa fille) et dans la $65^{\mathrm{e}} \mathrm{nv}$. (une vieille dévote est subitement "hors du sens" sous l'effet d'une gande frayeur).

38. Nouvelles $2,23,26,50$ et 70 pour les femmes et $9,10,13,50$ et 70 pour les hommes (dans les $12^{\mathrm{e}}$ et $31^{\mathrm{e}}$ nvs, les deux hommes meurent par punition de désirs illicites). Parmi les personnages ainsi "affectés" qui connaîtront la mort, un homme et une femme mourront "dans la joie": la chaste dame amoureuse du seigneur d'Avannes se meurt du combat que se livrent en elle l'amour et l'honneur et reçoit l'extrême onction "avecq telle joye comme celle qui est seure de son salut" (Saffredent, $26^{e}$ nv., p. 219); le gentilhomme de la $9^{\mathrm{e}} \mathrm{nv}$. ne peut épouser la femme qu'il aime, et la sait promise à un homme plus riche que lui: il meurt de joie lors d'une ultime étreinte (p. 52 - cf. supra, n. 33).

39. Soit en religion, soit par un volontaire retrait du monde ou par incarcération pénale: nvs 19, 22,24 et 40 .

40. Voici la répartition de ce réseau isotopique, sans égard à ses points de jonction avec les occurrences de "fantaisie": les devisants masculins considèrent que les hommes et les femmes connaissent le plus souvent, parmi les affections de l'âme, le désespoir, mais constatent que les hommes entrent un peu plus souvent en fureur et que les femmes perdent un peu plus le sens. Quant aux devisantes, elles insistent particulièrement sur la fureur comme cause de désordre chez les hommes (plus fréquente encore que le désespoir et la perte 
du sens) et insinuent que la mélancolie ne semble pas les affecter de façon significative tandis qu'elles identifient le désespoir et la perte du sens comme les désordres qui touchent le plus souvent les femmes.

Dans les nouvelles et commentaires énoncés par des hommes:

$\begin{array}{llll}\text { Fureur } & \text { Mélancolie } & \text { Désespoir } & \hat{E} \text { tre “hors du sens" } \\ 4 \text { hommes } & 2 \text { hommes } & 8 \text { hommes } & 2 \text { hommes } \\ 2 \text { femmes } & 3 \text { femmes } & 9 \text { femmes } & 4 \text { femmes }\end{array}$

Dans les nouvelles et commentaires énoncés par des femmes:

$\begin{array}{llll}\text { Fureur } & \text { Mélancolie } & \text { Désespoir } & \text { Etre "hors du sens" } \\ 12 \text { hommes } & 1 \text { homme } & 9 \text { hommes } & 8 \text { hommes } \\ 2 \text { femmes } & 3 \text { femmes } & 7 \text { femmes } & 6 \text { femmes }\end{array}$

En rapport avec les nouvelles où le réseau entre en cooccurrence avec la fantaisie:

$\begin{array}{llll}\text { Fureur } & \text { Mélancolie } & \text { Désespoir } & \text { Être "hors du sens" } \\ 3 \text { hommes } & 2 \text { hommes } & 5 \text { hommes } & 1 \text { homme } \\ 4 \text { femmes } & 4 \text { femmes } & 4 \text { femmes } & 1 \text { femme }\end{array}$

41. Il s'agit là d'une acception attestée de la notion de fantaisie. Voir l'Exposition moralle du Roman de la Rose, attribuée à Marot, p. 148 de l'édition des Oeuvres de Clement Marot de G. Defaux: "peult estre que ledict aucteur ne jectoit pas seulement son penser \& fantasie sus le sens litteral, ains plustost attiroit son espritau sens allegoric \& moral, comme l'un disant et entendant l'aultre."

42. Produite pour "vous apprendre à confesser que la nature des femmes et des hommes est de soy encline à tout vice, [. . . ]; et pour vous abbatre l' audace que vous prenez, quant on en dit à vostre honneur, je vous en vais montrer un exemple qui est très veritable."

43. Une autre femme et trois hommes subiront les affres d'une "fantasie" amoureuse: ils ne connaîtront cependant le désespoir et la mélancolie que dans la mesure où ils n' accéderont pas à la fruition de leurs désirs: il s'agit de la duchesse de Bourgogne ( $70^{\circ} \mathrm{nv}$.), du gentilhomme italien amoureux de Poline $\left(19^{\mathrm{e}} \mathrm{nv}\right.$.), du duc de Florence $\left(12^{\mathrm{e}} \mathrm{nv}\right.$.) et de Jean Pietre (50 nv.).

44. En complément à l'affirmation de cet envahissement plus rapide, chez la femme, de l'affection amoureuse, et en confirmation du topos des émotions et attitudes extrêmes des femmes, Géburon affirmera: "la passion la plus aveuglante, c'est l' amour, et la personne la plus aveuglée, c'est la femme, qui n'a pas la force de conduire un si grand faiz" (p. 397).

45. Dans une moindre mesure, Oisille remet également en cause le jugement de l'un de ses compagnons, lorsqu' au devis de la $56^{\mathrm{e}} \mathrm{nv}$., elle dit à Saffredent: "Vous avez toujours les plus faulses opinions que je vis jamais; car il vous semble que toutes les femmes soient de vostre complexion" (p. 351).

46. Assertion qui semble confortée par les paroles de Géburon (p. 133), et encore par Parlamente (p. 221). 
47. Pour une construction similaire, mais qui semble moins péjorative, voir Dagoucin à Ennasuitte, p. 341: "je vous supplie oster ceste opinion de vostre teste [. . .]."

48. Parallèlement au cynisme de Saffredent qui ne voit en l'honneur des femmes" qu'hypocrisie et dissimulation, Longarine expose qu'en certains cas, la dissimulation peut être adjuvante de la vertu (52 $\mathrm{nv} .$, p. 335$)$.

49. “'Ignorant les premières causes': jeux d'énigmes dans l'Heptaméron," dans L'Heptaméron de Marguerite de Navarre: Actes de la journée d'études Marguerite de Navarre, 19 octobre 1991, réunies et présentées par Simone Perrier (Paris, Université de Paris VII, 1992), pp. 81 83.

50. Les mêmes symptomes caractérisent Jehan Piètre, qui meurt toutefois de grande faiblesse, dans une grande effusion de sang ( $50^{\complement}$ nv., p. 325$)$.

51. Longarine, p. 340 et Oisille, p. 352.

52. Parlamente à l'égard de Dagoucin (discours indirect, p. 48). Dans le même sens, notons cet emploi "masculin" de "fantaisie" dans les devis: Géburon, devis de la 63 nv. (p. 382): "si une damoiselle m'avoit laissé tant enduré pour elle, je demanderais grande recompense, ou j'en retireroys ma fantaisye."

53. M. Bideaux, p. 125.

54. Que cette analyse du comportement féminin reçoive la caution de certaines nouvelles racontées par des femmes ne prouve pas qu'elle soit, de ce fait, récusée. Si le récit de Longarine illustrant combien les femmes "sont aisées à tromper, quant elles mectent leur fantaisye à la jalousye" ( $8^{\mathrm{e}}$ nv., p. 43) concourt implicitement à l'accréditation de la thèse d'Hircan et de Saffredent sur les erreurs de jugement causées par la fantaisie féminine, des phénomènes similaires surviennent en d'autres lieux du recueil, qui pourraient sembler confirmer la "doctrine" adverse, mais contribuent beaucoup plus à nuancer le propos, à établir des consensus par des variations sur le thème de la fragilité humaine, et à instaurer l'instabilité du sens. Ainsi, un récit d'Hircan vient illustrer l'énoncé de Parlamente affirmant, dans le devis de la $26^{\mathrm{e}}$ nv. (p. 221), que "nul n' [a] dommage" du péché d'orgueil des femmes, tandis que le plaisir des hommes "gist à deshonnorer les femmes" et leur "honneur à tuer les hommes en guerre"; Hircan raconte en effet comment un fils devenu incestueux, par omission et complaisance de sa mère, ne "desiroit autre chose que, après la joyssance de s'amye, s'en aller à la guerre” (30 nv., p. 231).

55. Nouvelles et devis confondus, les occurrences de "fantaisie" sont plus fréquemment liées au désir chez les hommes (nvs 12,15,19,43,47,50; devis 8 et 63, comparativement aux nvs 35,70 , et aux devis 53 et 56 chez les femmes) alors qu'elles accompagnent les erreurs de raisonnement et de jugement chez les femmes (nvs 8, 10; devis 42 et 21 pour une seule "fantaisie et fausse opinion" masculine, nv. 70). Mentionnons également que les devisants insinuent que la femme capitule par dépit, désespoir ou jalousie plutôt que par amour: voir Longarine, p. 127; Dagoucin, p. 314, et Simontault, p. 419.

56. Voir l'affirmation de Simontault selon laquelle "il y a eu plus d'hommes trompez par les femmes, que par les hommes" (p. 356), une affirmation qui rappelle les réflexions de Nature dans le Roman de la Rose, qui fait figurer parmi les puissances trompeuses la déesse Vénus 
ainsi que les subtilités, malices et menteries de femmes. La femme déçoit et abuse l'homme comme miroir, maladie ou mirage peuvent le faire, dans la mesure où elle peut être à l'origine des illusions, mensonges et "aultres visions merveilleuses / Soient doulces ou douloureuses/Quel'on voit advenir soubdaines/C'est assavoirs'elzsontforaines/Ou sans plus en la fantasie" (18640).

57. Voir les leçons contradictoires de Saffredent dans la $26^{\mathrm{e}} \mathrm{nv}$., réitérant par cette contradiction la suspicion à l'égard de l'honneur féminin qui ne serait, selon lui, qu'hypocrisie et dissimulation - moyens que Longarine rapportait à "la doctrine de Jehan de Mehun" (amorce de la $25^{\mathrm{c}}$ nv., p. 203) — qui font apparaître ici la "sage" comme étant la plus folle. Entre les indicateurs orientant le sens exemplaire du conte et le commentaire subséquent, qui superpose à la première moralité une seconde leçon (p. 220), Saffredent rompt avec la règle voulant que, généralement, ce sont les auditeurs d'une nouvelle qui en réévaluent le sens en proposant d'autres hypothèses sur l'exemplarité du fait. La révision de l'exemplarité de la nouvelle conforterait ainsi la doctrine de Saffredent sur l' "honneur féminin," au point que l'histoire de la "sage" illustre le comble de la dissimulation chez une femme qui s' abuse elle-même sur ses motivations profondes.

58. Dans le devis de la $26^{e}$ nv., toujours, Hircan réaffirme "la leçon de Jean de Meung," en rappelant "l'existence d'une loi de Nature dont il ne croit guère qu'elle pourrait être vicieuse" (M. Bideaux, p. 205).

59. Raison conseillait en effet à Christine, horrifiée à la lecture des Lamentations de Matheolus, se méprisant elle-même "et le sexe féminin tout entier, comme si la Nature avait enfanté des monstres," de juger des choses selon son expérience: "tu rejettes ce que tu sais en toute certitude pour te rallier à une opinion que tu ne crois, que tu ne connais et ne fondes que sur l'accumulation des préjugés d'autrui." Plus loin encore, au sujet du traité Du Secret des femmes, qui énumère les défauts des fonctions corporelles féminines, Raison conseille à Christine: "L'expérience de ton propre corps nous dispensera d'autres preuves. [Et] parce que les femmes peuvent savoir par expérience que certaines choses dans ce livre n'ont aucune réalité et qu'elles sont de pures bêtises, elles peuvent en déduire que les autres points qu'il expose sont autant de mensonges patents" (Cité des Dames, texte traduit et présenté par Thérèse Moreau et Éric Hicks (Paris, Stock, 1986), pp. 38-39, 53).

60. Voir par exemple, Saffredent, p. 265: "Et, si nous voulons dire la verité, il n'y a nul de nous qui n'ait experimenté ceste furieuse follye," qu'est l'amour.

61. Entre autres occurrences, signalons Hircan, p. 220-221: "quant à la chasteté de cueur, je croy qu'elle [ma femme] et moy sommes enfans d'Adam et Eve; parquoy, en bien nous mirant, n'aurons besoing de couvrir nostre nudité de feulles, mais plustost confesser nostre fragilité."

62. À cet égard, Oisille est la plus éloquente lorsqu'elle raconte ( $70^{\circ} \mathrm{nv}$., pp. 400-401) le dépit de la duchesse de Bourgogne qui, dédaignée par l'amoureux de la Dame du Vergier, "devint plus charnelle que les pourceaulx et plus cruelle que les lyons." Elle entre ensuite dans un "furieux desespoir [qui] la poussa tant, que, oubliant qu'elle estoit femme qui debvoit estre priée et refuser, princesse qui debvoit estre adorée [. . .] print le cueur d'un homme transporté pour descharger le feu qui estoit importable [et] se delibera de ne regarder craincte ny honte, mais luy declarer sa fantaisie [. . .]." 
63. Alors que l'honneur de la femme consisterait à savoir se vaincre elle-même: voir Parlamente (p. 294) et Longarine (p. 295).

64. Ne mentionnons ici que quelques exemples de ces causes spécifiquement féminines des désordres de l'âme chez les héroïnes des contes 21, 23, 50,51, 70 (récits de devisantes) et $14,16,26,30,35,43,60,72$ (récits de devisants). Nous avons déjà vu les cas de la "sage" dame de Pampelune atteinte de mélancolie ( $26^{\mathrm{C}} \mathrm{nv}$.) et de Jambique, dont la colère se mue en fureur au moment où son identité est découverte ( $43^{\mathrm{e}} \mathrm{nv}$.). Mais c'est la $23^{\mathrm{e}}$ nouvelle qui illustre le mieux notre propos, puisque fureur, désespoir et perte du sens y sont étroitement liés à l'honneur de l'héroïne, épouse d'un gentilhomme du Perigord, abusée par un cordelier: "La damoiselle, qui toute sa vye avoit aymé son honneur, entra en ung tel desespoir, que, oblyant toute humanité et nature de femme, le supplia à genoux de la venger de ceste grande injure" (p. 190); elle "se trouva si troublée, en l'assault de ce desespoir fondé sur l'enormité et gravité du peché, surl'amour du mary et l'honneur du lynaige, qu'elle estima la mort trop plus heureuse que la vie. Et, vaincue de sa tristesse, tumba en tel desespoir [.. .] fut du tout allienée du sens commung, obliant sa propre nature. Allors, vaincue de la douleur, poulsée du desespoir, hors de la congnoissance de Dieu et de soy-mesmes, comme femme enragée et furieuse, print une corde de son lict et de ses propres mains s'estrangla" (pp. 190-191). 